\title{
Design and analysis of mechanisms for decentralized joint replenishment
}

\author{
Kemal Güler ${ }^{\mathrm{a}}$, Evren Körpeoğlu ${ }^{\mathrm{b}}$, Alper Şen ${ }^{\mathrm{c}, *}$ \\ a Department of Economics, Faculty of Economic \& Administrative Sciences, Anadolu University, Eskisehir 26470, Turkey \\ b@WalmartLabs, San Bruno, CA 94066, USA \\ ${ }^{\mathrm{c}}$ Department of Industrial Engineering, Bilkent University, Bilkent, Ankara 06800, Turkey
}

\section{A R T I C L E I N F O}

\section{Article history:}

Received 3 November 2015

Accepted 11 November 2016

Available online 17 November 2016

\section{Keywords:}

Game Theory

Inventory

Joint replenishment

Economic Order Quantity model

Mechanism design

\begin{abstract}
A B S T R A C T
We consider jointly replenishing multiple firms that operate under an EOQ like environment in a decentralized, non-cooperative setting. Each firm's demand rate and inventory holding cost rate are private information. We are interested in finding a mechanism that would determine the joint replenishment frequency and allocate the joint ordering costs to these firms based on their reported stand-alone replenishment frequencies (if they were to order independently). We first provide an impossibility result showing that there is no direct mechanism that simultaneously achieves efficiency, incentive compatibility, individual rationality and budget-balance. We then propose a general, two-parameter mechanism in which one parameter is used to determine the joint replenishment frequency, another is used to allocate the order costs based on firms' reports. We show that efficiency cannot be achieved in this two-parameter mechanism unless the parameter governing the cost allocation is zero. When the two parameters are same (a single parameter mechanism), we find the equilibrium share levels and corresponding total cost. We finally investigate the effect of this parameter on equilibrium behavior. We show that properly adjusting this parameter leads to mechanisms that are better than other mechanisms suggested earlier in the literature in terms of fairness and efficiency.
\end{abstract}

(c) 2016 Elsevier B.V. All rights reserved.

\section{Introduction}

The classical Economic Order Quantity (EOQ) model is a wellknown and studied model in inventory management literature. The core of this model is the trade-off between inventory holding costs and setup costs associated with production, transportation or procurement. In the simplest form of the model, a firm faces deterministic demand with a constant rate, pays a setup cost for each replenishment order and incurs inventory holding costs for each unit of inventory it carries per unit of time. Minimizing setup and inventory holding costs gives the famous formula for the optimal order quantity. Since the first study (Harris, 1913), there has been a vast amount of literature on EOQ model, its extensions and the more general lot sizing problem. The interested reader is referred to Jans and Degraeve (2008) for a recent review.

A major cost saving opportunity in this setting is to consolidate orders for different items (or locations). By carefully coordinating

\footnotetext{
* Corresponding author. Fax: +90 3122664054.

E-mail addresses: kemalgoler@anadolu.edu.tr (K. Güler),

ekorpeoglu@walmartlabs.com (E. Körpeoğlu), alpersen@bilkent.edu.tr (A. Şen).
}

the replenishment of multiple items that may incur a joint setup, one can exploit the economies of scale of ordering jointly and reduce setup costs, inventories or both. This problem is known as Joint Replenishment Problem (JRP) and there is a growing literature in this area since 1960s. See Khouja and Goyal (2008) and Aksoy and Erengüç (1988) for two important reviews of research on this problem. The basic assumption in this literature is that the items or locations that are replenished jointly are also controlled centrally. However, this may not be always true. With intense and increasing pressure to reduce costs, independent, and sometimes competing firms may also be interested in jointly replenishing their inventories. For example, recently, BMW started an auto-parts purchasing partnership with one of its main competitors, Daimler, to procure more than 10 parts together and looking for ways to expand this partnership. BMW hoped to generate cost savings of around 100 million Euros annually through this venture (Gilbert, 2010). The advent of the Internet and B2B exchanges made collaborative purchasing and replenishment easier than ever and led to large scale and successful purchasing consortiums or groups. A recent review article states that collaboration is one of the most important trends and research opportunities in supply chain management (Speranza, 2016). 


\subsection{Related work}

Decentralized joint replenishment has attracted attention in literature only recently and studies until now investigate how the total savings (or total costs) should be allocated among participants using cooperative game theory. Meca, Timmer, GarciaJurado, and Borm (2004) propose a coordination scheme where the players only share their independent order frequencies prior to joint replenishment. Their allocation mechanism distributes the total setup cost among the players in proportion to the square of their order frequencies. They show that this allocation is in the core of the game. Fiestras-Janeiro, García-Jurado, Meca, and Mosquera (2015) study the case where the warehouse space for each player is limited, but the inventory holding costs are negligible. Timmer, Chessa, and Boucherie (2013) extend the work of Meca et al. (2004) for stochastic demand and suggest two coordination strategies.

When minor setup costs associated with each ordered item are also present, it may not be optimal to order every item with every replenishment. In fact, the structure of optimal policy is not known. For this problem, Hartman and Dror (2007) show that the game with a specific group of items has a core, whenever these items need to be ordered together on the same schedule to minimize total costs. Anily and Haviv (2007) focus on near optimal power-of-two policies for this problem, and show the existence and example of a core allocation. Zhang (2009) generalizes these results for the case of a sub-modular joint setup cost function and orders passing through a warehouse that may carry inventory. Minner (2007) uses bargaining models to study the collaboration between firms in a similar joint replenishment setting. For a recent review of research that uses cooperative game theory in inventory theory, see Fiestras-Janeiro, Garcia-Jurado, Meca, and Mosquera (2011).

In this paper, we follow a non-cooperative approach for the joint replenishment problem. Bauso, Giarre, and Presenti (2008) consider a periodic inventory model where each firm needs to determine the order quantities in each period to satisfy its demand. The demand in each period is different but known in advance. The fixed order cost is shared among multiple firms that order in the same period. They show the existence of pure strategy Nash equilibria and propose a consensus protocol that reaches to one of these equilibria. In Meca, Garcia-Jurado, and Borm (2003), each firm reports an order frequency (that may be different from its true order frequency) and the joint order frequency is determined to minimize the total joint costs based on these reports. Each firm incurs holding cost individually and pays a share of the joint replenishment cost in proportion to the squares of reported order frequencies. It is shown that this rule entails significant misreporting and inefficiency. It is shown that the game has multiple equilibria, in one of which none of the firms participate in joint replenishment. If the firms are sufficiently homogeneous, there also exists a (unique) "constructive equilibrium”, i.e., an equilibrium in which all firms participate in joint replenishment.

Körpeoğlu, Șen, and Güler (2012) follow a more direct approach using a two stage game. They assume that there is an intermediary that coordinates the replenishment activity. In Stage 1, each firm decides whether to participate in joint replenishment by agreeing to pay a minimum contribution or to replenish independently. In Stage 2, each participating firm submits a contribution to the intermediary. Then, the intermediary determines the minimum cycle time that can be financed with these contributions. It is shown that all firms participate in equilibrium and only those firms with the highest adjusted demand rates pay more than the minimum contribution. Körpeoğlu, Sen, and Güler (2013) study the private information version of the game in Körpeoğlu et al. (2012). It is shown that the privacy of information eliminates free-riding but contributions are not as high yielding higher aggregate costs.

\subsection{Contributions}

In this paper, we study the mechanism design problem for the joint replenishment of decentralized firms which have private information about their demand rates and inventory holding cost rates. We first study a direct mechanism where each firm reports its independent frequency and a joint replenishment frequency and the allocation of the joint order costs between the firms are decided based on these reports. We show that a direct mechanism which satisfies the efficiency, incentive compatibility and individual rationality constraints cannot satisfy the budget-balance constraint, i.e., a truth telling direct mechanism cannot finance the joint replenishment for efficient cycle times. Next, we generalize the mechanism suggested by Meca et al. (2003). While the mechanism in Meca et al. (2003) determines the joint order frequency and the order cost allocation both based on the squares of the reported stand-alone order frequencies, we use a general formulation in which two separate parameters govern these decisions. For this two-parameter mechanism, we show that the joint frequency is always lower than the efficient frequency unless the order cost is allocated uniformly. We then study the one-parameter mechanism, where these two parameters are equal to each other. We find the conditions necessary for a constructive equilibrium and characterize this equilibrium. We also provide necessary conditions for convexity at the equilibrium point. We analyze the comparative statics of the one-parameter model and show that using smaller values of this single parameter leads to better mechanisms in terms of fairness and efficiency.

\section{The model and preliminaries}

We consider a stylized EOQ environment with a set of firms $N=\{1, \ldots, n\}$. Demand rate for firm $i$ is constant and deterministic at $\beta_{i}$ per unit of time. Inventory holding cost per unit time for firm $i$ is $\gamma_{i}$ per unit. We denote the adjusted demand rate of firm $i$ as $\alpha_{i}=\gamma_{i} \beta_{i}$. We assume that adjusted demand rates are strictly positive, $\alpha_{i}>0$ for all $i \in N$ to rule out trivial replenishment environments where either the demand rate or the holding cost rate is zero. Major ordering cost is fixed at $\kappa$ per order regardless of order size. Minor ordering costs (ordering costs associated with firms included in an order) are assumed to be zero. We assume that the outside supplier that replenishes the orders has infinite capacity. The firms aim to minimize their long-run average costs over time and backorders are not allowed.

In any setting, the objective is to minimize the total cost rate, denoted by $C$, i.e., the sum of replenishment cost rate $(R)$ and holding cost rate $(H): C=R+H$. The decision variable can be taken as order cycle time, $t$, or order frequency, $f=1 / t$ (number of orders per time unit). We take frequency as the decision variable in the sequel.

Vectors are denoted by lower-case letters in bold typeface. For an endogenous variable $X$, by $X_{M}^{a}$ we refer to the value of $X$ when the set of firms is $M$ and replenishment operations are governed by $a \in\{c, d, 2 p, 1 p\}$, where $c$ stands for centralized, $d$ stands for decentralized (or independent) replenishment, $2 p$ stands for two-parameter mechanism and $1 p$ stands for the single-parameter mechanism. For instance, $C_{M}^{c}$ is the total cost of the firms in $M$ when replenishment is centralized. When the set $M$ is a singleton, e.g., $M=\{i\}$, we use $X_{i}^{a}$ instead of $X_{\{i\}}^{a}$. Exceptions to this notation are used for $f_{i}$, the optimal frequency of the decentralized replenishment for firm $i$ and for $f_{*}$, the optimal frequency of centralized replenishment. 


\subsection{Independent (decentralized) replenishment}

When the replenishment of the items is controlled by firms operating independently, the problem is the well-known EOQ model. Firm $i$ 's total cost rate $\left(C_{i}\right)$ is the sum of replenishment cost rate $\left(R_{i}\right)$ and the holding cost rate $\left(H_{i}\right)$ can be found:

$C_{i}(f)=R_{i}(f)+H_{i}(f)=\kappa f+\frac{\alpha_{i}}{2 f}$.

It can be easily found that firm i's optimal frequency is $f_{i}=$ $\sqrt{\alpha_{i} / 2 \kappa}$ (Zipkin, 2000, Ch. 3). With this frequency, optimal replenishment cost rate and optimal inventory holding cost rate are equal at $R_{i}^{d}=H_{i}^{d}=\kappa f_{i}$. The aggregate total cost rate for all firms under independent replenishment is therefore $C_{N}^{d}=\sum_{i \in N} 2 \kappa f_{i}$.

\subsection{Centralized joint replenishment}

When all firms cooperate, they order with a joint order frequency to achieve the efficiency. Meca et al. (2004) show that when there are no minor setup costs, it is optimal for all firms to be replenished in each cycle and this leads to a common order frequency. Denoting the joint order frequency by $f$, the total cost under cooperation is given by

$C_{N}(f)=R_{N}(f)+H_{N}(f)=\kappa f+\frac{\sum_{i \in N} \alpha_{i}}{2 f}=\kappa f+\kappa \frac{\sum_{i \in N} f_{i}^{2}}{f}$.

Using the first order condition, we obtain the efficient frequency as $f_{*}=\left(f_{1}^{2}+\ldots+f_{n}^{2}\right)^{1 / 2}$. The efficient total cost is then $C_{N}^{c}=2 \kappa f_{*}$.

We use the proportional rule of Meca et al. (2004) which simply allocates the order costs based on the proportion of adjusted demand rate of firm $i$ to the sum of adjusted demand rates. This rule is in the core of the cooperative game. With this proportional rule, the cost share of firm $i$ is $\alpha_{i} /\left(\alpha_{1}+\cdots+\alpha_{n}\right)$. Since, $f_{i}^{2}=\alpha_{i} /(2 \kappa)$, we can rewrite the cost share as $f_{i}^{2} /\left(f_{1}^{2}+\cdots+f_{n}^{2}\right)$. Thus the cost of firm $i$ under cooperation is given by

$C_{i}^{c}=\frac{2 \kappa f_{i}^{2}}{\sqrt{f_{1}^{2}+\cdots+f_{n}^{2}}}$

\section{Mechanism design for the joint replenishment problem}

We consider the design of a mechanism for the joint replenishment problem. A mechanism is a specification of how economic decisions should be taken for a set of players who are privately informed about their preferences based on the messages they provide to an intermediary. Mechanism design problem usually consists of three steps. In step 1, the mechanism is designed. In step 2 , the players accept or reject the mechanism. If a firm rejects the mechanism, it gets an exogenously specified reservation utility. In step 3, the players play the game specified by the mechanism and economic outcomes and payoffs for each player are determined. A mechanism is efficient if it maximizes the sum of player's payoffs. A truth-telling strategy is a strategy in which the player reports true information about his preference, regardless of the value of his preference. A mechanism is incentive compatible if for any player, truth-telling is a dominant-strategy. A mechanism is individually rational if for any player the mechanism leads to a payoff that is at least as much as his reservation utility. A direct mechanism is a mechanism where each player sends a message regarding his preference.

We consider designing a mechanism for the joint replenishment problem. We assume that each firm's adjusted demand rate, $\alpha_{i}$ for firm $i$, is observable, but not verifiable. Each firm's adjusted demand rate, or consequently, its optimal independent order frequency $f_{i}$ (since $f_{i}=\sqrt{\frac{\alpha_{i}}{2 \kappa}}$ and $\kappa$ is common knowledge) can be considered as its type. We assume that the types are independent draws. In addition to the firms in $N=\{1, \ldots, n\}$, we introduce the player $n+1$ that will be responsible for the replenishment. The mechanism will select an outcome or a joint frequency $f$ as a result of the players' reports of their types. Each firm's utility can be represented in the quasi-linear form as follows:

$u_{i}\left(f, f_{i}, p_{i}\right)=-\kappa \frac{f_{i}^{2}}{f}-p_{i}$,

where the first term is the firm $i$ 's value for alternative $f$ or its inventory holding cost rate. The second term is the payment by the firm to the mechanism. The player $n+1$ 's utility can also be represented in quasi-linear form:

$u_{n+1}\left(f, p_{n+1}\right)=-\kappa f-p_{n+1}$,

where the first term is the replenishment costs incurred and the second term is the payment of player $n+1$. Each firm's reservation utility is equal to its independent optimal cost rate, $C_{i}^{d}=2 \kappa f_{i}$ for firm $i$. Player $n+1$ 's reservation utility is zero.

We consider a direct mechanism, therefore firms report their independent replenishment frequencies. In this case, a mechanism will be defined by an outcome rule which specifies the joint replenishment frequency and a payment rule which specifies the payments by each player as a function of the reported independent replenishment frequencies. An efficient mechanism for this problem should select the optimal frequency of the centralized problem $f_{*}=\left(f_{1}^{2}+\cdots+f_{n}^{2}\right)^{1 / 2}$ as the joint replenishment frequency leading to total costs that is equal to the total costs for the centralized problem, i.e., $2 \kappa f_{*}$. A common requirement for a mechanism in this setting is budget-balance. This condition requires that the sum of payments from firms in $N$ through the mechanism should finance the joint setup or ordering cost incurred by player $n+1$. The main question in mechanism design is whether there is a direct mechanism for the joint replenishment problem that is efficient, incentive compatible, individually rational and budget-balanced. The answer to this question is unfortunately negative which follows from Myerson and Satterthwaite (1983) who show that the there are no mechanisms that satisfy these four properties simultaneously for bilateral trading and Williams (1999) which generalizes this result for multi-firm general settings where the firms have quasi-linear utilities.

Given this impossibility result for direct mechanisms, we will revisit the mechanism suggested by Meca et al. (2003) for competitive environments and explore its generalizations. According to this mechanism, each firm $i$ reports its optimal independent frequency $\hat{s}_{i}$ (which can be different from the true optimal independent frequency $f_{i}$ ) without knowing the choices of other firms. Let $\hat{\boldsymbol{s}}=\left(\hat{s}_{1}, \hat{s}_{2}, \ldots, \hat{s}_{n}\right)$ be the vector of reported optimal independent frequencies. If only one firm has chosen a positive $\hat{s}_{i}$ in this step, then all firms order alone and incur their stand-alone optimal cost rate. Otherwise, all firms that reported a positive independent frequency order jointly. In this case, the joint frequency is selected as $\sqrt{\sum_{j \in N} \hat{s}_{j}^{2}}$ (i.e., outcome rule). These firms incur inventory holding costs based on this joint frequency. The joint setup costs are allocated to these firms based on the proportional rule that Meca et al. (2003) suggest for the cooperative setting. Namely, firm $i$ is allocated $\hat{s}_{i}^{2} / \sum_{j \in N} \hat{s}_{j}^{2}$ of the joint replenishment costs. As a result, a firm $i$ that reports a positive independent frequency gets a total cost

$\frac{1}{2} \alpha_{i} \sqrt{\sum_{j \in N} \hat{s}_{j}^{2}}+\frac{\kappa \hat{s}_{i}^{2} \sqrt{\sum_{j \in N} \hat{s}_{j}^{2}}}{\sum_{j \in N} \hat{s}_{j}^{2}}=\frac{\kappa f_{i}^{2}}{\sqrt{\sum_{j \in N} \hat{s}_{j}^{2}}}+\frac{\kappa \hat{s}_{i}^{2} \sqrt{\sum_{j \in N} \hat{s}_{j}^{2}}}{\sum_{j \in N} \hat{s}_{j}^{2}}$,

where the first term is the incurred inventory holding costs and the second term is the allocation of the joint order costs. Finally, 
all firms that reported $\hat{s_{i}}=0$ in the first step order alone. In most of their analysis, Meca et al. (2003) focus on constructive equilibria where $\hat{s}_{i}>0$ for all $i \in N$. Since firms with $\hat{s}_{i}=0$ do not participate in joint replenishment, in the absence of minor setup costs, any equilibrium that is not constructive will clearly suffer from inefficiency. Meca et al. (2003) show that this mechanism can entail significant misreporting and lead to inefficient joint decisions. Therefore, in the next two sections, we study generalizations of this mechanism.

\section{Two-parameter mechanisms}

In the previous section we showed that there is no truth-telling direct mechanism that can achieve efficiency, individual rationality and budget-balance simultaneously. In this section we consider a general class of mechanisms and investigate their ability to reach an efficient and fair outcome. We again assume that adjusted demand rates, thus independent frequencies are observable by all firms, but not verifiable. We assume that each firm reports a frequency denoted by $\hat{s}_{i}$ for firm $i$ and a mechanism determines the joint order frequency and the allocation of the setup cost based on these reports. We consider a two-parameter mechanism where one parameter $(\xi)$ governs the joint order frequency decision and another parameter $(\theta)$ governs the allocation decision. In particular, the joint frequency under the two parameter mechanism is $\left(\hat{s}_{1}^{\xi}+\cdots+\hat{s}_{n}^{\xi}\right)^{1 / \xi}$, and replenishment setup cost share of firm $i$ is $\hat{s}_{i}^{\theta} /\left(\hat{s}_{1}^{\theta}+\cdots+\hat{s}_{n}^{\theta}\right)$. Since we allocate all of the setup cost using the parameter $\xi$, the budget-balance condition is trivially satisfied for this mechanism.

Using these values we can easily find the total cost rate $C_{i}^{2 p}$ for firm $i$ as

$C_{i}^{2 p}(\hat{\mathbf{s}})=\kappa f_{i}^{2}\left(\sum_{j \in N} \hat{s}_{j}^{\xi}\right)^{-\frac{1}{\xi}}+\frac{\kappa \hat{s}_{i}^{\theta}\left(\sum_{j \in N} \hat{s}_{j}^{\xi}\right)^{\frac{1}{\xi}}}{\sum_{j \in N} \hat{s}_{j}^{\theta}}$.

The first term on the right hand side of (5) is the average inventory holding cost. The second term is the time averaged order cost that is allocated to firm $i$. Note that the cost of firm $i$ depends on its reported frequency as well as its rivals'. Therefore, we have a non-cooperative game where each firm's strategy is its reported frequency and we can use Nash equilibrium as a solution concept.

In order to find the best response correspondence of firm $i$ to the strategies of other firms, we obtain the first order condition. Denoting the equilibrium strategy vector as $\boldsymbol{s}=\left(s_{1}, . ., s_{n}\right)$, the first order condition at the equilibrium is given by:

$$
\begin{aligned}
& \left.\frac{\partial C_{i}^{2 p}(\hat{\mathbf{s}})}{\partial \hat{s}_{i}}\right|_{\hat{\mathbf{s}}=\mathbf{s}}=-\kappa f_{i}^{2} s_{i}^{\xi-1}\left(\sum_{j \in N} s_{j}^{\xi}\right)^{-\frac{1}{\xi}-1} \\
& -\kappa \theta s_{i}^{2 \theta-1}\left(\sum_{j \in N} s_{j}^{\xi}\right)^{\frac{1}{\xi}}\left(\sum_{j \in N} s_{j}^{\theta}\right)^{-2} \\
& +\kappa \theta s_{i}^{\theta-1}\left(\sum_{j \in N} s_{j}^{\xi}\right)^{\frac{1}{\xi}}\left(\sum_{j \in N} s_{j}^{\theta}\right)^{-1} \\
& +\kappa s_{i}^{\theta+\xi-1}\left(\sum_{j \in N} s_{j}^{\xi}\right)^{\frac{1}{\xi}-1}\left(\sum_{j \in N} s_{j}^{\theta}\right)^{-1}=0 .
\end{aligned}
$$

We can simplify this equation by
$\kappa^{-1} s_{i}^{1-\theta}\left(\sum_{j \in N} s_{j}^{\theta}\right)^{2}\left(\sum_{j \in N} s_{j}^{\xi}\right)^{1-\frac{1}{\xi}}$ which yields

$$
\begin{aligned}
& f_{i}^{2} s_{i}^{\xi-\theta}\left(\sum_{j \in N} s_{j}^{\theta}\right)^{2}\left(\sum_{j \in N} s_{j}^{\xi}\right)^{-\frac{2}{\xi}}=\theta\left(\sum_{j \in N} s_{j}^{\theta}\right)\left(\sum_{j \in N} s_{j}^{\xi}\right) \\
& +s_{i}^{\xi}\left(\sum_{j \in N} s_{j}^{\theta}\right)-\theta s_{i}^{\theta}\left(\sum_{j \in N} s_{j}^{\xi}\right) .
\end{aligned}
$$

By rearranging the terms, we obtain

$$
\begin{aligned}
f_{i}^{2}= & \theta s_{i}^{\theta-\xi}\left(\sum_{j \in N} s_{j}^{\theta}\right)^{-1}\left(\sum_{j \in N} s_{j}^{\xi}\right)^{\frac{2+\xi}{\xi}}+s_{i}^{\theta}\left(\sum_{j \in N} s_{j}^{\theta}\right)^{-1}\left(\sum_{j \in N} s_{j}^{\xi}\right)^{\frac{2}{\xi}} \\
& -\theta s_{i}^{2 \theta-\xi}\left(\sum_{j \in N} s_{j}^{\xi}\right)^{\frac{2+\xi}{\xi}}\left(\sum_{j \in N} s_{j}^{\theta}\right)^{-2} .
\end{aligned}
$$

This implicit function gives the equilibrium reported frequencies $s_{i}$, but no further simplification is possible and a closed form solution for the equilibrium is not available. However, we can determine the performance (with respect to its ability to reach the efficient solution) of the two-parameter mechanism by the following proposition.

Proposition 1. The ratio of the efficient frequency and the equilibrium frequency under the two-parameter mechanism is given by:

$$
\begin{aligned}
& \left(\frac{\left(\sum_{i \in N} f_{i}^{2}\right)^{1 / 2}}{\left(\sum_{i \in N} s_{i}^{\xi}\right)^{1 / \xi}}\right)^{2}=1+\left(\sum_{i \in N} s_{i}^{\theta}\right)^{-2} \theta \\
& \quad \times\left(2 \sum_{i \neq j} s_{i}^{\theta} s_{j}^{\theta}+\sum_{i \neq j} s_{i}^{\theta+\xi} s_{j}^{\theta-\xi}+\sum_{i \neq j, j \neq k} s_{i}^{\theta} s_{j}^{\xi} s_{k}^{\theta-\xi}\right) .
\end{aligned}
$$

Since we are interested in only constructive equilibria where $s_{i}$ $>0$ for all $i \in N$, Proposition 1 shows that unless $\theta=0$, the efficient joint frequency is always larger than the joint frequency in the constructive equilibrium (if it exists) which in turn implies that cooperative solutions would give smaller costs for all firms. This is formally given in the following corollary.

Corollary 1. For the two-parameter mechanisms, the joint frequency in a constructive equilibrium is always less than the efficient frequency unless the order cost allocation parameter $\theta=0$, i.e., the order cost is allocated uniformly.

However, $\theta=0$ is only a necessary condition for efficiency. There is no guarantee that an equilibrium under a uniform cost allocation exists. Next, we present an example with $\xi=2$ where the equilibrium does not exist in general.

\section{A special case: $(\boldsymbol{\xi}, \boldsymbol{\theta})=(\mathbf{2}, \mathbf{0})$}

We consider a two-parameter mechanism with joint frequency parameter as $(\xi=2)$ and sharing parameter as $(\theta=0)$ which corresponds to a uniform sharing (replenishment cost share of firm $i=1 / n$ ). This is an important special case since on one hand efficiency can be obtained only if $\theta=0$ as shown in Corollary 1 and on the other hand $\xi=2$ leads to efficient joint replenishment frequency if the firms were to report their true stand-alone frequencies. 
In this case, the payoff for firm $i$ is:

$$
\begin{aligned}
C_{i}^{2 p}(\hat{\mathbf{s}}) & =\frac{1}{n} \kappa\left(\sum_{j \in N} \hat{s}_{j}^{2}\right)^{\frac{1}{2}}+\kappa f_{i}^{2}\left(\sum_{j \in N} \hat{s}_{j}^{2}\right)^{-\frac{1}{2}} \\
& =\frac{\kappa}{n}\left(\left(\sum_{j \in N} \hat{s}_{j}^{2}\right)^{\frac{1}{2}}+n f_{i}^{2}\left(\sum_{j \in N} \hat{s}_{j}^{2}\right)^{-\frac{1}{2}}\right) .
\end{aligned}
$$

First order condition for optimal response is:

$$
\begin{aligned}
\left.\frac{\partial C_{i}^{1 p}(\hat{\mathbf{s}})}{\partial \hat{s}_{i}}\right|_{\hat{\mathbf{s}}=\mathbf{s}} & =\frac{\kappa s_{i}}{n}\left(\left(\sum_{j \in N} s_{j}^{2}\right)^{-\frac{1}{2}}-n f_{i}^{2}\left(\sum_{j \in N} s_{j}^{2}\right)^{-\frac{3}{2}}\right) \\
& =\frac{\kappa s_{i}}{n}\left(\sum_{j \in N} s_{j}^{2}\right)^{-\frac{3}{2}}\left(s_{i}^{2}+\sum_{j \neq i} s_{j}^{2}-n f_{i}^{2}\right)=0 .
\end{aligned}
$$

We obtain the best responses as $s_{i}^{2}=n f_{i}^{2}-\sum_{j \neq i} s_{j}^{2}$ and derive the equilibrium frequency as

$$
\begin{aligned}
& \sum_{j \in N} s_{j}^{2}=n \sum_{j \in N} f_{j}^{2}-(n-1) \sum_{j \in N} s_{j}^{2} \\
\Rightarrow & \sum_{j \in N} s_{j}^{2}=\sum_{j \in N} f_{j}^{2} \\
\Rightarrow & \sqrt{\sum_{j \in N} s_{j}^{2}}=\sqrt{\sum_{j \in N} f_{j}^{2}} \\
\Rightarrow & f_{\xi}=f_{*},
\end{aligned}
$$

which is equal to the cooperative joint frequency. However, note that the best responses are $s_{i}^{2}=n f_{i}^{2}-\sum_{j \neq i} s_{j}^{2}$ which leads to $f_{i}=$ $\sqrt{\sum_{j \in N} s_{j}^{2}} / n$. Therefore in order to have an equilibrium, all firms should have the same stand-alone frequency. Otherwise, there is no constructive equilibrium and each firm replenishes independently.

Since further analysis of the two-parameter mechanisms is not tractable, in the next section, we explore one parameter mechanisms in detail.

\section{One-parameter mechanisms}

In this section, we consider a single parameter mechanism where we set the value of the parameters for determining the joint order frequency and allocating the ordering costs equal to each other. This is done primarily due to the fact that the analysis of two-parameter mechanisms is intractable. The one-parameter mechanisms admit an easier mathematical and numerical analysis. In addition, the only mechanism in the literature, the mechanism suggested in Meca et al. (2003) is a special version of the one-parameter policy where the single parameter takes on the value 2 .

When we assume that $\theta=\xi$, the resulting cost function for a given vector of reports $\hat{\mathbf{s}}$ is

$C_{i}^{1 p}(\hat{\mathbf{s}})=\kappa f_{i}^{2}\left(\sum_{j \in N} \hat{s}_{j}^{\xi}\right)^{-\frac{1}{\xi}}+\kappa \hat{s}_{i}^{\xi}\left(\sum_{j \in N} \hat{s}_{j}^{\xi}\right)^{\frac{1}{\xi}-1}$.

In this case, Eq. (6) simplifies to

$f_{i}^{2}=\xi\left(\sum_{j \in N} s_{j}^{\xi}\right)^{\frac{2}{\xi}}+s_{i}^{\xi}(1-\xi)\left(\sum_{j \in N} s_{j}^{\xi}\right)^{\frac{2}{\xi}-1}$ and (7) can be written as

$$
\begin{aligned}
& \left(\frac{\left(\sum_{i \in N} f_{i}^{2}\right)^{1 / 2}}{\left(\sum_{i \in N} s_{i}^{\xi}\right)^{1 / \xi}}\right)^{2} \\
& =1+\left(\sum_{i \in N} s_{i}^{\xi}\right)^{-2} \xi\left(2 \sum_{i \neq j} s_{i}^{\xi} s_{j}^{\xi}+(n-1) \sum_{i \in N} s_{i}^{2 \xi}+2(n-2) \sum_{i \neq j} s_{i}^{\xi} s_{j}^{\xi}\right) \\
& =1+\left(\sum_{i \in N} s_{i}^{\xi}\right)^{-2} \xi(n-1)\left(\sum_{i \in N} s_{i}^{2 \xi}+2 \sum_{i, j \in N, i \neq j} s_{i}^{\xi} s_{j}^{\xi}\right) \\
& =1+\xi(n-1) .
\end{aligned}
$$

Denoting the joint frequency in equilibrium $f_{\xi}=\left(\sum_{i \in N} s_{i}^{\xi}\right)^{1 / \xi}$, we obtain

$$
\frac{f_{*}}{f_{\xi}}=\sqrt{\xi(n-1)+1},
$$

which shows that the deviation of the equilibrium joint frequency from the efficient joint frequency depends only on the parameter $\xi$ and $n$. In particular, $f_{*}>f_{\xi}$ for all $\xi>0$ and $f_{*} / f_{\xi}$ is an increasing function of $\xi$. This means that the one parameter mechanisms are never perfectly efficient in general, but their efficiency improves as $\xi$ gets smaller.

In order to further characterize the equilibrium, we first obtain the best response function for firm $i$. The expression in (9) can be written as:

$$
\left(\frac{\sum_{j \in N} f_{j}^{2}}{\xi(n-1)+1}\right)^{\frac{\xi}{2}}=\sum_{j \in N} s_{j}^{\xi}
$$

Therefore, the best response of firm $i$ is given by

$$
s_{i}^{\xi}=\left(\frac{\sum_{j \in N} f_{j}^{2}}{\xi(n-1)+1}\right)^{\frac{\xi}{2}}-\sum_{j \in N \backslash\{i\}} s_{j}^{\xi}, \text { for } i=1, \ldots, n .
$$

Clearly, there can be equilibria in which a firm reports 0 and stays out of the joint replenishment. As is stated before and also in Meca et al. (2003), when one or more firms stay out of the joint replenishment, we are sure that the total costs will be higher than the optimal centralized costs (since there are only major setup costs). Therefore and since our focus is efficiency, we are mainly interested in constructive equilibria where each firm reports a positive frequency.

We can use the best response functions (11) in (8) and rearrange the terms to get the following equality for the equilibrium reports:

$s_{i}^{\xi}=\frac{\xi \sum_{j \in N} f_{j}^{2}-((n-1) \xi+1) f_{i}^{2}}{((n-1) \xi+1)(\xi-1)}\left(\frac{\sum_{j \in N} f_{j}^{2}}{(n-1) \xi+1}\right)^{\xi / 2-1}$.

If $\xi>1$, the argument in (13) is positive if and only if $\xi \sum_{j \in N} f_{j}^{2}-$ $((n-1) \xi+1) f_{i}^{2}>0$. On the other hand, if $\xi<1$, the argument in (13) is positive if and only if $\xi \sum_{j \in N} f_{j}^{2}-((n-1) \xi+1) f_{i}^{2}<0$. Since these conditions have to be satisfied for all firms, we can formalize these conditions in the following proposition.

Proposition 2. The necessary condition for a constructive equilibrium for the one-parameter mechanism is given by

$$
\frac{\max _{j \in N} f_{j}^{2}}{\sum_{j \in N} f_{j}^{2}}<\frac{\xi}{(n-1) \xi+1},
$$

if $\xi>1$, and 
$\frac{\min _{j \in N} f_{j}^{2}}{\sum_{j \in N} f_{j}^{2}}>\frac{\xi}{(n-1) \xi+1}$,

if $\xi<1$.

Proposition 2 shows that the constructive equilibrium can exist only if firms' stand-alone optimal frequencies are close to each other. Note that these conditions are only necessary conditions. In order to show that the solution in (13) is in fact the equilibrium, we need to show that the payoff function is convex. We provide the conditions for this in the following proposition.

Proposition 3. The cost function is convex at (13) and the solution in (13) is a Nash equilibrium if and only if

$\xi \sum_{j \in N} f_{j}^{2}-(\xi-2)((n-1) \xi+1) f_{i}^{2} \geq 0$, for all $i=1, \ldots, n$.

A consequence of this result is that for $\xi>3$, we do not have convexity at the equilibrium point regardless of the frequency distribution and for $\xi \leq 2$ we always have convexity. Therefore, when $\xi \leq 2$, we can state the conditions in Proposition 2 also as sufficient conditions for constructive equilibrium.

We are now ready to express the costs incurred by each firm in equilibrium under the single parameter joint replenishment mechanism. The cost of firm $i$ in equilibrium can be found by using the equilibrium reports $\mathbf{S}=\left(s_{1}, \ldots, s_{n}\right)$ as follows:

$C_{i}^{1 p}(\mathbf{s})=\kappa f_{i}^{2}\left(\sum_{j \in N} s_{j}^{\xi}\right)^{-\frac{1}{\xi}}+\kappa s_{i}^{\xi}\left(\sum_{j \in N} s_{j}^{\xi}\right)^{\frac{1}{\xi}-1}$

In equilibrium, using (11) and (13):

$$
\begin{aligned}
C_{i}^{1 p}(\mathbf{s}) & =\kappa f_{i}^{2}\left(\frac{\sum_{j \in N} f_{j}^{2}}{(n-1) \xi+1}\right)^{-\frac{1}{2}} \\
& +\kappa \frac{\xi \sum_{j \in N} f_{j}^{2}-((n-1) \xi+1) f_{i}^{2}}{((n-1) \xi+1)(\xi-1)}\left(\frac{\sum_{j \in N} f_{j}^{2}}{(n-1) \xi+1}\right)^{-\frac{1}{2}} .
\end{aligned}
$$

Taking the terms to $\left(\frac{\sum_{j \in N} f_{j}^{2}}{(n-1) \xi+1}\right)^{-\frac{1}{2}}$ parenthesis and rearranging the terms gives the equilibrium cost of firm $i$ as:

$$
\begin{aligned}
C_{i}^{1 p}(\mathbf{s})= & \kappa\left(\frac{\xi \sum_{j \in N} f_{j}^{2}+(\xi-2)((n-1) \xi+1) f_{i}^{2}}{((n-1) \xi+1)(\xi-1)}\right) \\
& \times\left(\frac{\sum_{j \in N} f_{j}^{2}}{(n-1) \xi+1}\right)^{-\frac{1}{2}} .
\end{aligned}
$$

Summing over all the firms, we obtain the total cost as

$$
\begin{aligned}
C_{N}^{1 p}(\mathbf{s}) & =\sum_{j \in N} C_{j}^{1 p}(\mathbf{s}) \\
& =\kappa\left(\frac{\xi n+(\xi-2)((n-1) \xi+1)}{\xi-1}\right)\left(\frac{\sum_{j \in N} f_{j}^{2}}{\xi(n-1)+1}\right)^{\frac{1}{2}},
\end{aligned}
$$

and the cost ratio of firm $i$ is given by

$\frac{C_{i}^{1 p}(\mathbf{s})}{C_{N}^{1 p}(\mathbf{s})}=\kappa\left(\frac{\xi \sum_{j \in N} f_{j}^{2}+(\xi-2)((n-1) \xi+1) f_{i}^{2}}{\xi n+(\xi-2)((n-1) \xi+1)}\right)\left(\sum_{j \in N} f_{j}^{2}\right)^{-1}$.

\section{A special case: $\xi=\mathbf{2}$}

A special case of our one-parameter mechanisms is the mechanism used in Meca et al. (2003) where the parameter is $\xi=2$. We revisit this mechanism as this is the only mechanism suggested in the literature and we would like pose this as a benchmark for different values of $\xi$.

In this case, the necessary and sufficient condition for a constructive equilibrium given in Proposition 2 simplifies to

$f_{i}^{2} \leq \frac{2}{2 n-3} \sum_{j \neq i} f_{j}^{2}$, for all $i=1,2, \ldots, n$,

as is also shown in Theorem 2 of Meca et al. (2003). The equilibrium joint frequency simplifies to:

$f_{\xi}=\frac{1}{\sqrt{2 n-1}} f_{*}<f_{*}$.

Since $f_{\xi}<f_{*}$, clearly this mechanism is inefficient and will lead to total cost more than the optimal centralized total costs.

The cost of firm $i$ in this case is:

$$
\begin{aligned}
C_{i}^{1 p} & =\kappa\left(\frac{2 \sum_{j \in N} f_{j}^{2}}{(2(n-1)+1)}\right)\left(\frac{\sum_{j \in N} f_{j}^{2}}{2(n-1)+1}\right)^{-\frac{1}{2}} \\
& =2 \kappa\left(\frac{\sum_{j \in N} f_{j}^{2}}{2(n-1)+1}\right)^{\frac{1}{2}},
\end{aligned}
$$

which shows that each firm has the same cost under joint replenishment regardless of their stand-alone frequencies or adjusted demand rates. This result shows that in addition to being inefficient, the mechanism in Meca et al. (2003) is also not desirable in terms of fairness.

\section{Impact of $\xi$ and comparative statics}

We now investigate how the equilibrium behavior and efficiency change as a function of $\xi$ and stand-alone frequencies. For this purpose we obtain the comparative statics for the game.

First remember that Eq. (10) states $\frac{f_{*}}{f_{\xi}}=\sqrt{\xi(n-1)+1}$, and therefore we know that the efficiency of the one parameter mechanism improves as $\xi$ gets smaller. One can also derive an expression for the difference between reported frequencies of two firms $i, k$ with $f_{i}>f_{k}$ as follows:

$s_{i}^{\xi}-s_{k}^{\xi}=\frac{f_{k}^{2}-f_{i}^{2}}{(\xi-1)}\left(\frac{\sum_{j \in N} f_{j}^{2}}{(n-1) \xi+1}\right)^{\xi / 2-1}$,

which shows that for $\xi>1$, we have $s_{i}<s_{k}$. Therefore, the firm with higher stand-alone frequency reports a lower frequency than a firm with lower stand-alone frequency. For $\xi<1$, the firm with higher stand-alone frequency reports a higher frequency. A similar expression can be derived for equilibrium cost of two firms as follows:

$C_{i}^{1 p}-C_{k}^{1 p}=\kappa\left(\frac{(\xi-2)\left(f_{i}^{2}-f_{k}^{2}\right)}{(\xi-1)}\right)\left(\frac{\sum_{j \in N} f_{j}^{2}}{(n-1) \xi+1}\right)^{-\frac{1}{2}}$.

Eq. (16) can be used to show that for $1<\xi<2, C_{i}^{1 p}<C_{k}^{1 p}$, i.e., the firm with higher stand-alone frequency has a lower equilibrium cost. For $\xi<1$ or $\xi>2$, the reverse is true and we have $C_{i}^{1 p}>C_{k}^{1 p}$. Therefore, from a fairness perspective, mechanisms with $\xi<1$ or $\xi>2$ are preferable to those with $1<\xi<2$.

It is also important to understand how a firm's equilibrium frequency report changes as its own true stand-alone frequency or its competitor's stand-alone frequency changes. We can derive the partial derivative of the equilibrium reported frequency of firm $i, s_{i}$ 

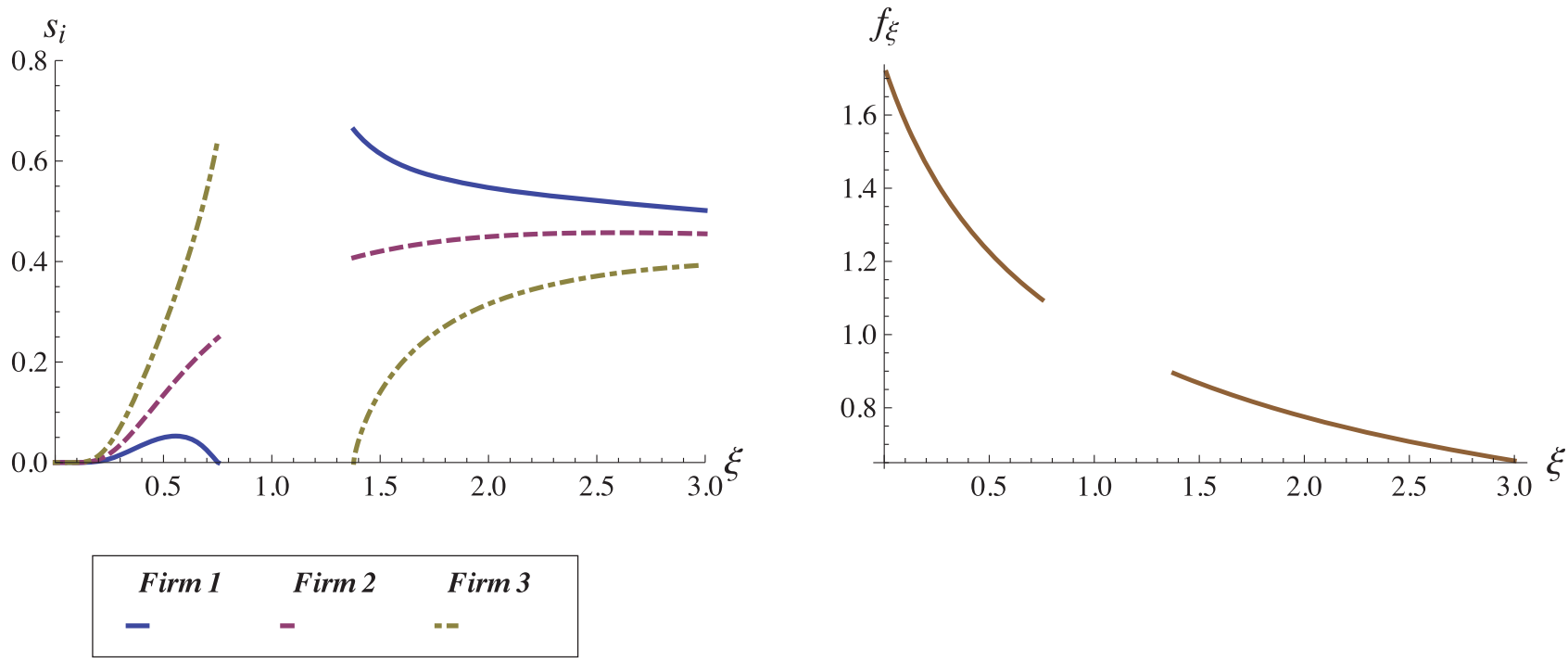

Fig. 1. Reported Frequencies and Equilibrium Joint frequency as a function of $\xi$ for $\left(f_{1}, f_{2}, f_{3}\right)=(0.95,1,1.05)$.

with respect to its own stand-alone frequency $f_{i}$ as follows:

$\frac{\partial s_{i}}{\partial f_{i}}=\frac{f_{i} s_{i}}{\xi}$

$\times\left(\frac{\left.\left(\xi^{2}-2((n-1) \xi+1)\right) \sum_{j \in N} f_{j}^{2}-(\xi-2)((n-1) \xi+1) f_{i}^{2}\right)}{\xi \sum_{j \in N} f_{j}^{2}-((n-1) \xi+1) f_{i}^{2}}\right)$

$\times\left(\sum_{j \in N} f_{j}^{2}\right)^{-1}$

Similarly, the partial derivative with respect to a rival firm $k$ 's true frequency is

$\frac{\partial s_{i}}{\partial f_{k}}=\frac{f_{k} s_{i}}{\xi}\left(\frac{\xi^{2} \sum_{j \in N} f_{j}^{2}-(\xi-2)((n-1) \xi+1) f_{i}^{2}}{\xi \sum_{j \in N} f_{j}^{2}-((n-1) \xi+1) f_{i}^{2}}\right)\left(\sum_{j \in N} f_{j}^{2}\right)^{-1}$.

Corresponding changes in equilibrium costs are given by the following

$\frac{\partial C_{i}^{1 p}}{\partial f_{i}}$

$=\kappa f_{i}\left(\frac{(\xi+2(\xi-2)((n-1) \xi+1)) \sum_{j \in N} f_{j}^{2}-(\xi-2)((n-1) \xi+1) f_{i}^{2}}{((n-1) \xi+1)^{1 / 2}(\xi-1)}\right)$

$$
\times\left(\sum_{j \in N} f_{j}^{2}\right)^{-\frac{3}{2}}
$$

$\frac{\partial C_{i}^{1 p}}{\partial f_{k}}=\kappa f_{k}\left(\frac{\xi \sum_{j \in N} f_{j}^{2}-(\xi-2)((n-1) \xi+1) f_{i}^{2}}{((n-1) \xi+1)^{1 / 2}(\xi-1)}\right)\left(\sum_{j \in N} f_{j}^{2}\right)^{-\frac{3}{2}}$.

One can also consider the effect of an additional firm, firm $n+1$, entering the joint replenishment, on the reported frequency of firm $i$. For brevity, we only consider the difference of the $\xi^{\text {th }}$ power of the reported frequencies.

$$
\begin{aligned}
s_{i}^{\xi}(N \cup\{n+1\})-s_{i}^{\xi}(N) & \\
= & \frac{\xi\left(\sum_{j \in N} f_{j}^{2}+f_{n+1}^{2}\right)-(n \xi+1) f_{i}^{2}}{(n \xi+1)(\xi-1)}\left(\frac{\sum_{j \in N} f_{j}^{2}+f_{n+1}^{2}}{n \xi+1}\right)^{\xi / 2-1} \\
& -\frac{\xi \sum_{j \in N} f_{j}^{2}-((n-1) \xi+1) f_{i}^{2}}{((n-1) \xi+1)(\xi-1)}\left(\frac{\sum_{j \in N} f_{j}^{2}}{(n-1) \xi+1}\right)^{\xi / 2-1} .
\end{aligned}
$$

Correspondingly, the change in equilibrium costs can be shown as follows

$$
\begin{aligned}
& C_{i}^{1 p}(N \cup\{n+1\})-C_{i}^{1 p}(N) \\
& =\kappa\left(\frac{\xi\left(\sum_{j \in N} f_{j}^{2}+f_{n+1}^{2}\right)+(\xi-2)(n \xi+1) f_{i}^{2}}{(n \xi+1)^{1 / 2}(\xi-1)}\right)\left(\sum_{j \in N} f_{j}^{2}+f_{n+1}^{2}\right)^{-\frac{1}{2}} \\
& -\kappa\left(\frac{\xi \sum_{j \in N} f_{j}^{2}+(\xi-2)((n-1) \xi+1) f_{i}^{2}}{((n-1) \xi+1)^{1 / 2}(\xi-1)}\right)\left(\sum_{j \in N} f_{j}^{2}\right)^{-\frac{1}{2}} .
\end{aligned}
$$

It is interesting to note that the expression in (22) can take positive or negative values meaning that adding a new firm to the joint replenishment program does not necessarily decrease an existing firm's total costs in a one-parameter mechanism. For example, this may happen when the new firm's standalone frequency is low and $\xi$ is less than 1 . In this case, expecting that the new firm will report a considerable frequency, existing firms decrease their reported frequencies resulting in lower joint frequency and higher total costs for all firms. In situations like these, it may be useful to reveal some information regarding the new entry's characteristics such that incumbent firms determine their reported frequencies accordingly and benefit from the new entry.

\section{Numerical examples}

We demonstrate some of the sensitivity results for the oneparameter mechanisms using numerical examples in this section. First, we demonstrate the effect of $\xi$ on equilibrium reported frequencies, joint frequency, individual costs and total costs in a test problem with three firms with $\left(f_{1}, f_{2}, f_{3}\right)=(0.95,1,1.05)$ in Figs. 1 and 2 as $\xi$ varies between 0 and 3. Note that the efficient joint frequency for this problem is $f^{*}=1.733$. Fig. 1 shows the equilibrium frequency reports and resulting joint frequency as a function of $\xi$. Notice that we have a region of $\xi$ for which there is no constructive equilibrium.

Corresponding costs (as a percentage of total efficient costs) for each firm and total costs are shown in Fig. 2. Since the equilibrium joint frequency approaches the efficient joint frequency as $\xi$ gets smaller, total costs also approaches to the efficient total costs in this direction. Also notice that left plot of Fig. 2 confirms our analytical finding in (16). In the first region of $\xi$ which contains 

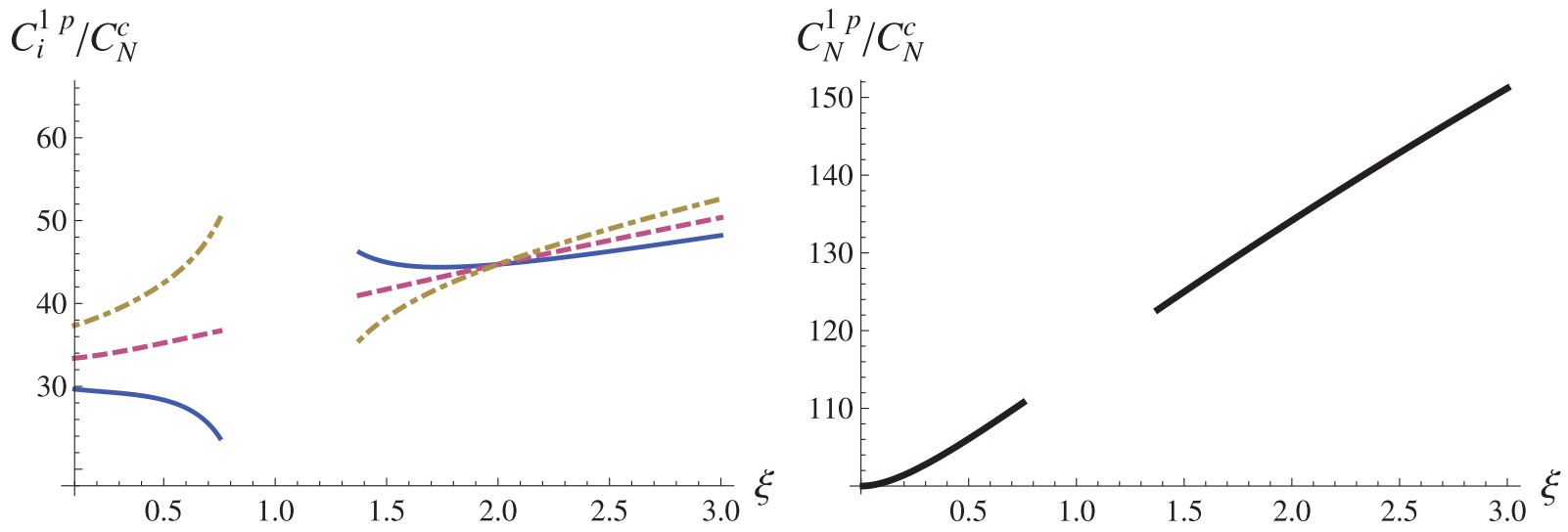

Fig. 2. Equilibrium individual costs and total cost as a percentage of efficient cost as a function of $\xi$ for $\left(f_{1}, f_{2}, f_{3}\right)=(0.95,1,1.05)$.
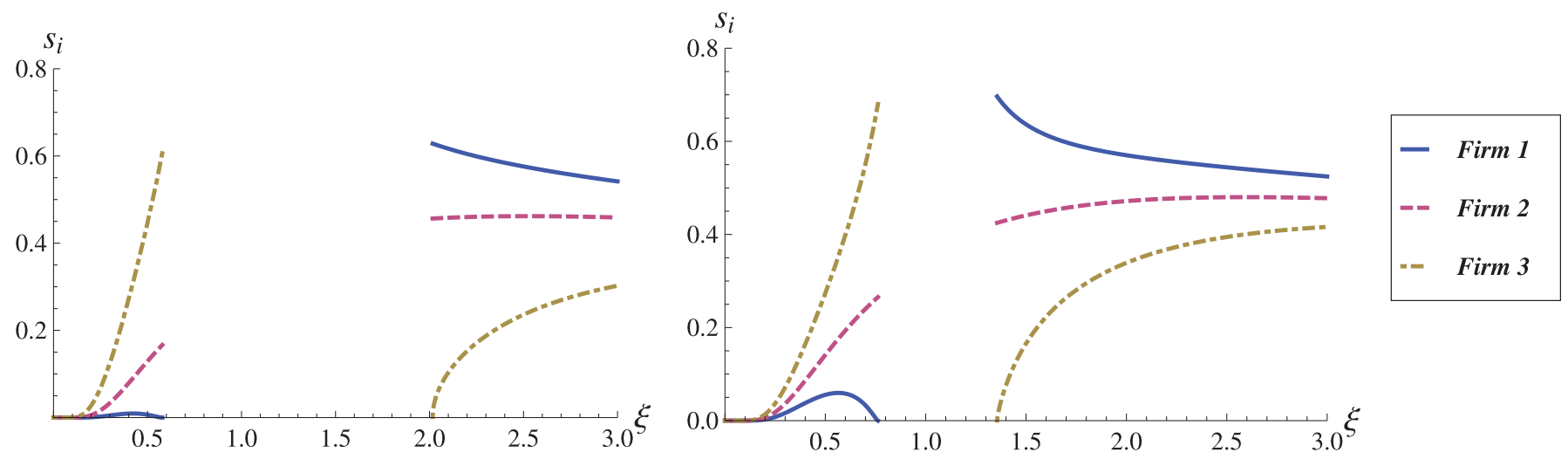

Fig. 3. Reported frequencies as a function of $\xi$ for $\left(f_{1}, f_{2}, f_{3}\right)=(0.9,1,1.1)$ and $\left(f_{1}, f_{2}, f_{3}\right)=(1,1.05,1.1)$.
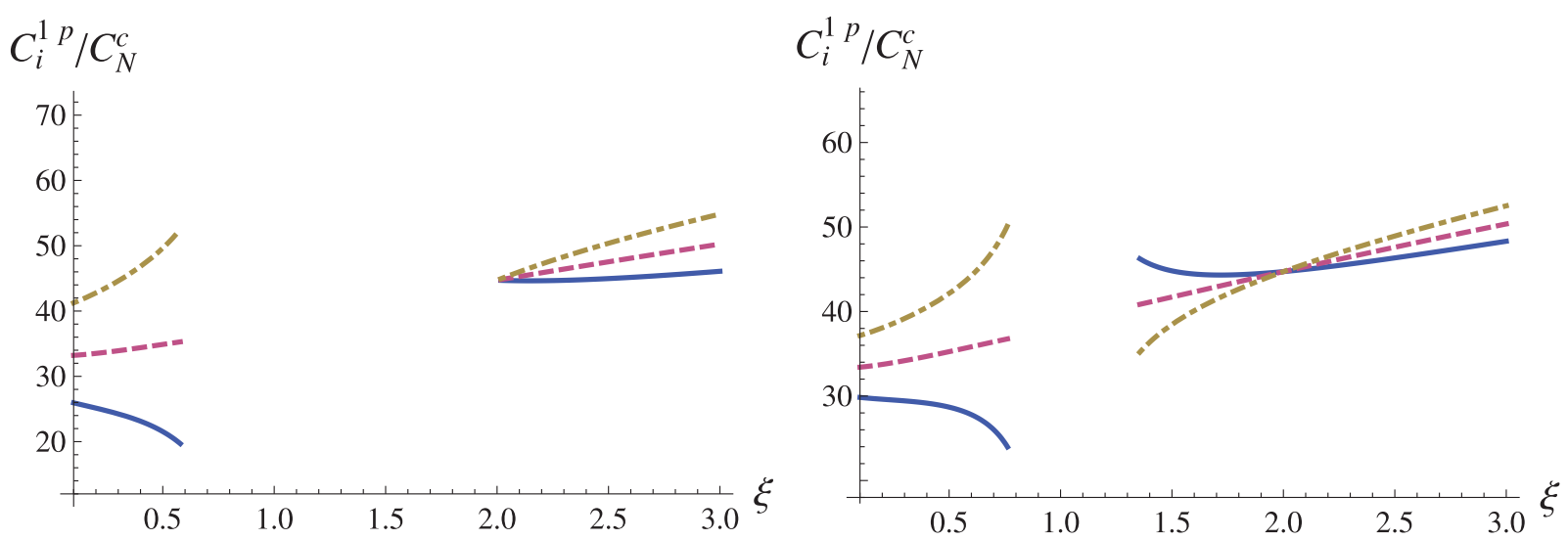

Fig. 4. Equilibrium firms costs as a percentage of efficient cost as a function of $\xi$ for $\left(f_{1}, f_{2}, f_{3}\right)=(0.9,1,1.1)$ and $\left(f_{1}, f_{2}, f_{3}\right)=(1,1.05,1.1)$.

constructive equilibrium $(\xi<1)$, the equilibrium cost of a higher stand-alone frequency (or higher adjusted demand rate) firm is always larger than the equilibrium cost of a firm with a lower standalone frequency. This simple sense of "fairness" is not guaranteed in the second region $(\xi>1)$.

Based on Eqs. (10), (15), and (16), and Figs. 1 and 2, using $\xi=2$ (as in Meca et al., 2003) is not desirable from an efficiency and fairness perspective. One needs to have $\xi<1$ for fairness. In addition, Proposition 2 and Eq. (15) implies that lower values of $\xi$ should be preferred for efficiency and to ensure a constructive equilibrium. The only downside of using very small values of $\xi$ seem to be the fact that the differences between reported frequencies are indistinguishable.

Figs. 3-5 show equilibrium reported frequencies, individual firm costs and total costs, respectively, for two other test problems: $\left(f_{1}, f_{2}, f_{3}\right)=(0.9,1,1.1)$ and $\left(f_{1}, f_{2}, f_{3}\right)=(1,1.05,1.1)$. The results are similar to the results for the first problem, except that the region for which no constructive equilibrium can be obtained expands (shrinks) as stand-alone frequencies get closer to (further away from) each other.

In Fig. 6, we compute the comparative statics given in (17) and (18) for the effects of own and rival's true replenishment frequency on a firm's reported frequency for the test problem with $\left(f_{1}, f_{2}, f_{3}\right)=(0.95,1,1.05)$. Fig. 6 shows that when $\xi<1$, the firm should report higher frequencies as its true frequency increases. This is in contrast to the second region of constructive equilibrium, where the firm report lower frequency as its true frequency increases. For the same problem, the comparative statics given in (19) and (20) are shown in Fig. 7. Fig. 7 shows that equilibrium cost for a firm is increasing in its own frequency and 

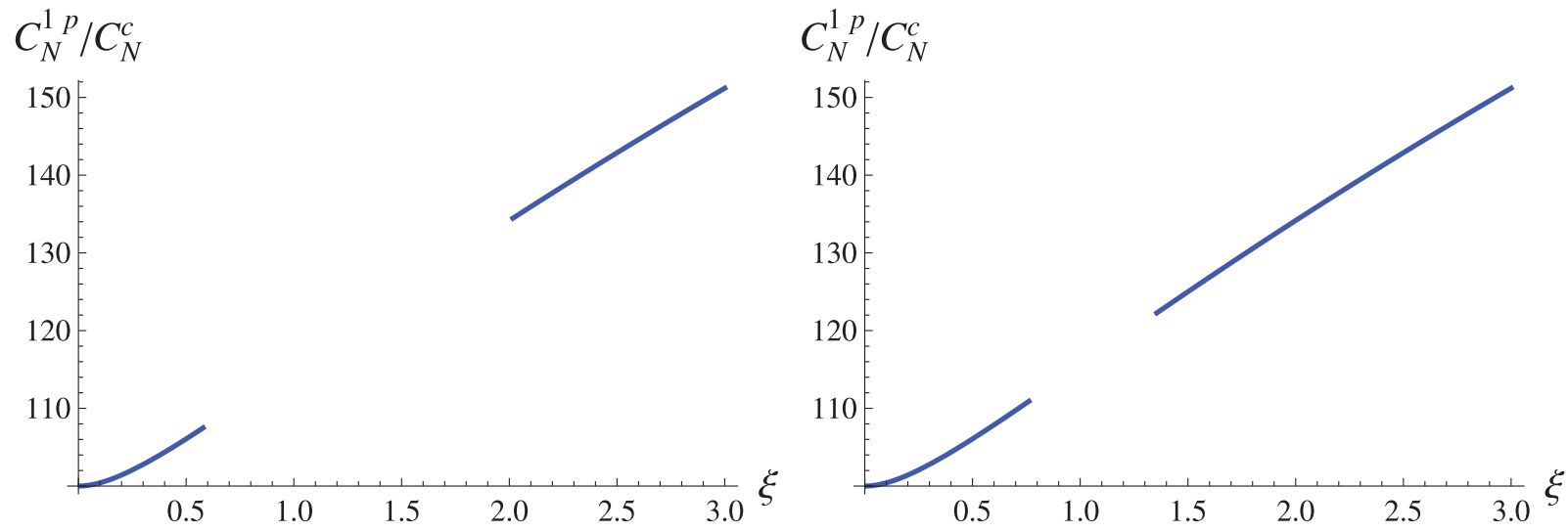

Fig. 5. Equilibrium total cost as a percentage of efficient cost as a function of $\xi$ for for $\left(f_{1}, f_{2}, f_{3}\right)=(0.9,1,1.1)$ and $\left(f_{1}, f_{2}, f_{3}\right)=(1,1.05,1.1)$.

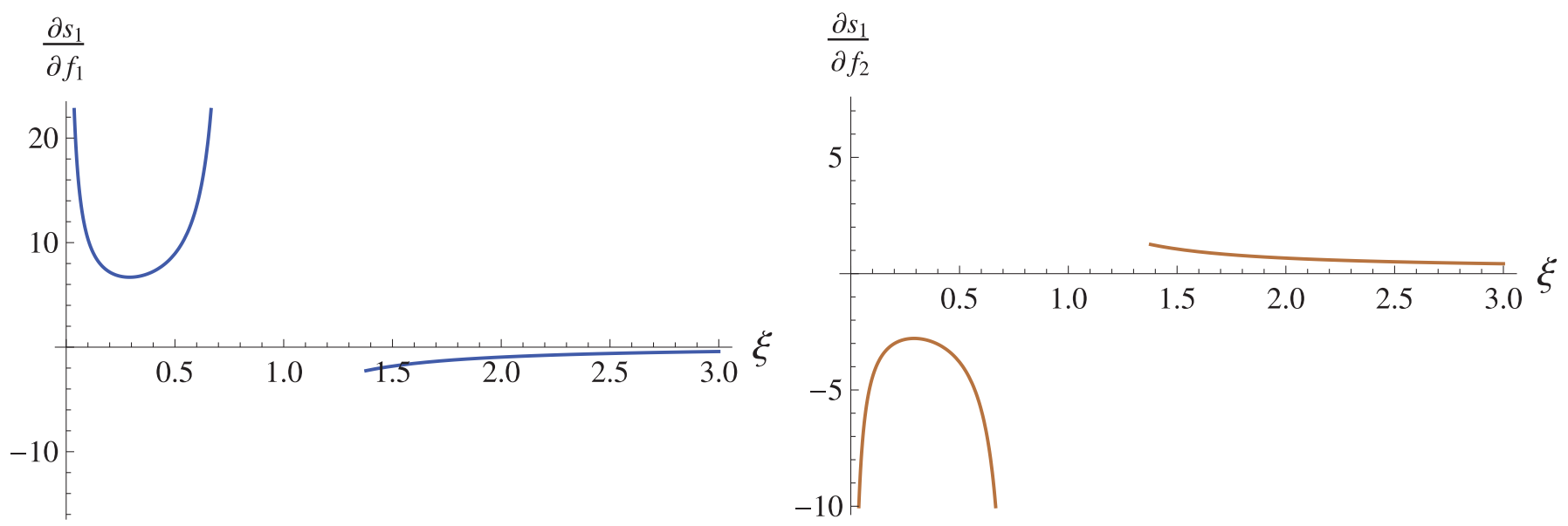

Fig. 6. Rate of change of firm 1's equilibrium reports with $f_{1}$ and $f_{2}$ as a function of $\xi$ for $\left(f_{1}, f_{2}, f_{3}\right)=(0.95,1,1.05)$.
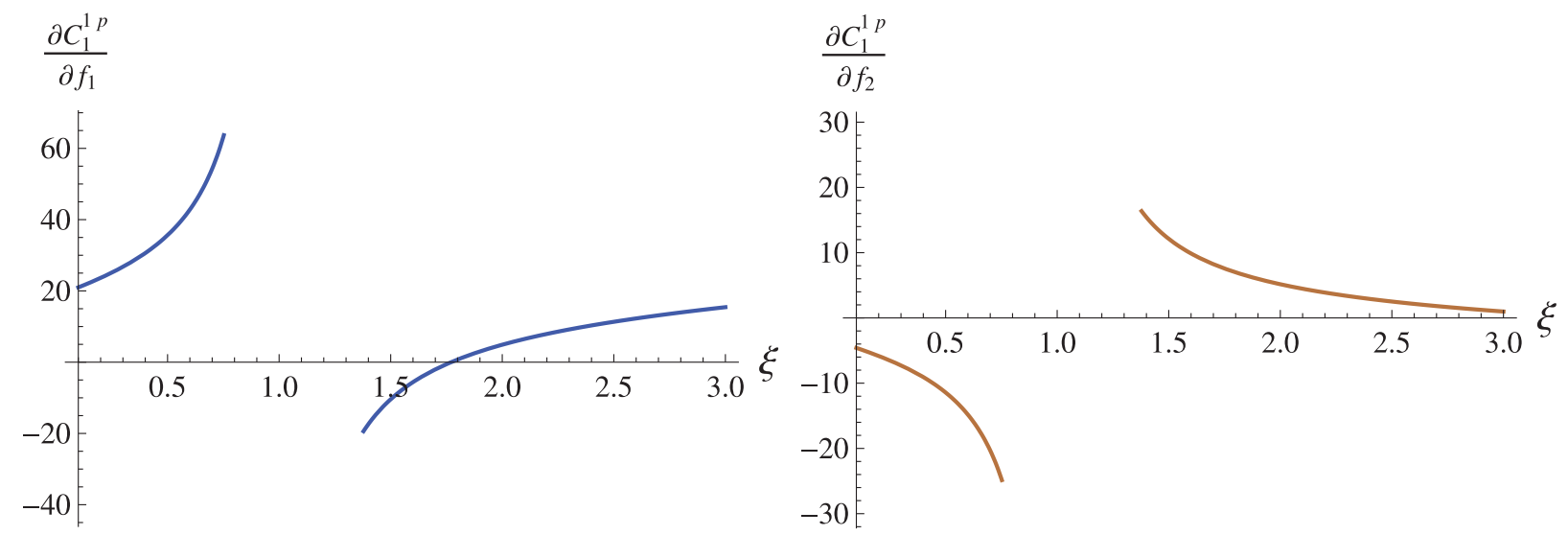

Fig. 7. Rate of change of firm 1's cost with $f_{1}$ and $f_{2}$ as a function of $\xi$ for $\left(f_{1}, f_{2}, f_{3}\right)=(0.95,1,1.05)$.

decreasing in its rival's frequency when $\xi<1$ and and the signs are reversed when $\xi>1$. The results in Figs. 6 and 7 confirm that using $\xi<1$ leads to a more desirable mechanism in terms of fairness.

\section{Conclusion}

In this paper, we consider jointly replenishing multiple, decentralized firms under an EOQ like environment. We assume that the adjusted demand rates are observable, but not verifiable and therefore investigate the use of direct and indirect mechanisms to determine a joint replenishment frequency and allocate setup costs. First, we show that there is no direct mechanism that is efficient, incentive compatible, individually rational, and budget-balanced. Hence, we explore specific mechanisms and investigate their ability to reach efficient and fair outcomes. In particular, we first study two-parameter mechanisms in which one parameter governs the joint frequency decision and the other governs the setup cost allocation. We show that it is not possible to achieve efficiency unless the setup costs are allocated uniformly. When these two parameters are equal, we derive conditions for the constructive equilibrium and characterize the equilibrium and comparative statics. We show that mechanisms with smaller values of this single parameter lead to more efficient outcomes and are more defendable in terms of fairness. 


\section{Acknowledgment}

The research supporting the final revision of this paper is undertaken during Kemal Güler's visit at Bilkent University supported by a TÜBITAK BIDEP 2236 Co-Circulation fellowship. He thanks TÜBITAK for financial support, colleagues at Bilkent University Industrial Engineering Department for their hospitality, and Bariş Ali, Betül, Elfe, and Sertuğ for their big hearts and warm Ankara memories.

\section{Appendix}

Proof of Proposition 1

Summing (6) over all $i \in N$ yields:

$$
\begin{aligned}
& \sum_{i \in N} f_{i}^{2}=\left(\sum_{i \in N} s_{i}^{\theta}\right)^{-1}\left(\sum_{i \in N} s_{i}^{\xi}\right)^{2 / \xi}\left(\theta \sum_{i \in N} s_{i}^{\xi} \sum_{i \in N} s_{i}^{\theta-\xi}+\sum_{i \in N} s_{i}^{\theta}\right. \\
& \left.-\theta \sum_{i \in N} s_{i}^{2 \theta-\xi} \sum_{i \in N} s_{i}^{\xi}\left(\sum_{i \in N} s_{i}^{\theta}\right)^{-1}\right) \\
& =\left(\sum_{i \in N} s_{i}^{\theta}\right)^{-2}\left(\sum_{i \in N} s_{i}^{\xi}\right)^{2 / \xi}\left(\theta \sum_{i \in N} s_{i}^{\xi} \sum_{i \in N} s_{i}^{\theta-\xi} \sum_{i \in N} s_{i}^{\theta}\right. \\
& \left.+\left(\sum_{i \in N} s_{i}^{\theta}\right)^{2}-\theta \sum_{i \in N} s_{i}^{2 \theta-\xi} \sum_{i \in N} s_{i}^{\xi}\right) \\
& =\left(\sum_{i \in N} s_{i}^{\theta}\right)^{-2}\left(\sum_{i \in N} s_{i}^{\xi}\right)^{2 / \xi}\left(\theta \left(2 \sum_{i \neq j} s_{i}^{\theta} s_{j}^{\theta}+\sum_{i \neq j} s_{i}^{\theta+\xi} s_{j}^{\theta-\xi}\right.\right. \\
& \left.\left.+\sum_{i \neq j, j \neq k} s_{i}^{\theta} s_{j}^{\xi} s_{k}^{\theta-\xi}\right)+\left(\sum_{i \in N} s_{i}^{\theta}\right)^{2}\right) \\
& =\left(\sum_{i \in N} s_{i}^{\xi}\right)^{2 / \xi}\left(( \sum _ { i \in N } s _ { i } ^ { \theta } ) ^ { - 2 } \theta \left(2 \sum_{i \neq j} s_{i}^{\theta} s_{j}^{\theta}\right.\right. \\
& \left.\left.+\sum_{i \neq j} s_{i}^{\theta+\xi} s_{j}^{\theta-\xi}+\sum_{i \neq j, j \neq k} s_{i}^{\theta} s_{j}^{\xi} s_{k}^{\theta-\xi}\right)+1\right) .
\end{aligned}
$$

Dividing both sides by $\left(\sum_{i \in N} s_{i}^{\xi}\right)^{2 / \xi}$ leads to the desired result.

\section{Proof of Proposition 3}

For the single parameter case the second derivative of the payoff function is as follows:

$$
\begin{aligned}
\left.\frac{\partial^{2} C_{i}^{1 p}(\hat{\mathbf{s}})}{\partial \hat{s}_{i}^{2}}\right|_{\hat{\mathbf{s}}=\mathbf{s}}= & -\kappa f_{i}^{2}(\xi-1) s_{i}^{\xi-2}\left(\sum_{j \in N} s_{j}^{\xi}\right)^{-\frac{1}{\xi}-1} \\
& -\kappa f_{i}^{2}(-1-\xi) s_{i}^{2 \xi-2}\left(\sum_{j \in N} s_{j}^{\xi}\right)^{-\frac{1}{\xi}-2} \\
& +\kappa \xi(\xi-1) s_{i}^{\xi-2}\left(\sum_{j \in N} s_{j}^{\xi}\right)^{\frac{1}{\xi}-1} \\
& +\kappa \xi(1-\xi) s_{i}^{2 \xi-2}\left(\sum_{j \in N} s_{j}^{\xi}\right)^{\frac{1}{\xi}-2}
\end{aligned}
$$

$$
\begin{aligned}
& +\kappa(1-\xi)(2 \xi-1) s_{i}^{2 \xi-2}\left(\sum_{j \in N} s_{j}^{\xi}\right)^{\frac{1}{\xi}-2} \\
& +\kappa(1-\xi)(1-2 \xi) s_{i}^{3 \xi-2}\left(\sum_{j \in N} s_{j}^{\xi}\right)^{\frac{1}{\xi}-3} .
\end{aligned}
$$

Factoring the expression, we obtain

$$
\begin{aligned}
& \left.\frac{\partial^{2} C_{i}^{1 p}(\hat{\mathbf{s}})}{\partial \hat{s}_{i}^{2}}\right|_{\hat{\mathbf{s}}=\mathbf{s}}=\kappa s_{i}^{\xi-2}\left(\sum_{j \in N} s_{j}^{\xi}\right)^{-\frac{1}{\xi}-3} \\
& \left(f_{i}^{2}\left(\sum_{j \in N} s_{j}^{\xi}\right)\left((1-\xi)\left(\sum_{j \in N} s_{j}^{\xi}\right)+(1+\xi) s_{i}^{\xi}\right)\right. \\
& \left.+(\xi-1)\left(\sum_{j \in N} s_{j}^{\xi}\right)^{\frac{2}{\xi}}\left(\sum_{j \neq i} s_{j}^{\xi}\right)\left(\xi\left(\sum_{j \in N} s_{j}^{\xi}\right)-(2 \xi-1) s_{i}^{\xi}\right)\right) .
\end{aligned}
$$

For convexity, the argument above should be non-negative. Using this, we get the following condition:

$$
\begin{aligned}
& (\xi-1)\left(\sum_{j \in N} s_{j}^{\xi}\right)^{\frac{2}{\xi}-1}\left(\sum_{j \neq i} s_{j}^{\xi}\right)\left(\xi\left(\sum_{j \in N} s_{j}^{\xi}\right)-(2 \xi-1) s_{i}^{\xi}\right) \\
& \geq f_{i}^{2}\left((\xi-1)\left(\sum_{j \in N} s_{j}^{\xi}\right)-(1+\xi) s_{i}^{\xi}\right) .
\end{aligned}
$$

Using (11) and (13) in the inequality, we get

$$
\begin{aligned}
& (\xi-1)\left(\left(\frac{\sum_{j \in N} f_{j}^{2}}{\xi(n-1)+1}\right)^{\frac{\xi}{2}}\right)^{\frac{2}{\xi}-1}\left(\left(\frac{\sum_{j \in N} f_{j}^{2}}{\xi(n-1)+1}\right)^{\frac{\xi}{2}}\right. \\
& \left.-\frac{\xi \sum_{j \in N} f_{j}^{2}-((n-1) \xi+1) f_{i}^{2}}{((n-1) \xi+1)(\xi-1)}\left(\frac{\sum_{j \in N} f_{j}^{2}}{(n-1) \xi+1}\right)^{\xi / 2-1}\right) \\
& \left(\xi\left(\frac{\sum_{j \in N} f_{j}^{2}}{\xi(n-1)+1}\right)^{\frac{\xi}{2}}\right. \\
& \left.-(2 \xi-1) \frac{\xi \sum_{j \in N} f_{j}^{2}-((n-1) \xi+1) f_{i}^{2}}{((n-1) \xi+1)(\xi-1)}\left(\frac{\sum_{j \in N} f_{j}^{2}}{(n-1) \xi+1}\right)^{\xi / 2-1}\right) \\
& \geq f_{i}^{2}\left((\xi-1)\left(\frac{\sum_{j \in N} f_{j}^{2}}{\xi(n-1)+1}\right)^{\frac{\xi}{2}}\right. \\
& \left.-(1+\xi) \frac{\xi \sum_{j \in N} f_{j}^{2}-((n-1) \xi+1) f_{i}^{2}}{((n-1) \xi+1)(\xi-1)}\left(\frac{\sum_{j \in N} f_{j}^{2}}{(n-1) \xi+1}\right)^{\xi / 2-1}\right) .
\end{aligned}
$$

Simplifying the terms yields

$$
\begin{aligned}
(\xi-1) & \left(\frac{((n-1) \xi+1) f_{i}^{2}-\sum_{j \in N} f_{j}^{2}}{((n-1) \xi+1)(\xi-1)}\right) \\
& \times\left(\frac{(2 \xi-1)((n-1) \xi+1) f_{i}^{2}-\xi^{2} \sum_{j \in N} f_{j}^{2}}{((n-1) \xi+1)(\xi-1)}\right) \\
\geq & f_{i}^{2}\left(\frac{(\xi+1)((n-1) \xi+1) f_{i}^{2}-(3 \xi-1) \sum_{j \in N} f_{j}^{2}}{((n-1) \xi+1)(\xi-1)}\right) .
\end{aligned}
$$

Next, we consider the cases for $\xi>1$ and $\xi<1$ separately since the equilibrium conditions for both cases are different. For 
$\xi>1$ the condition is:

$\left(((n-1) \xi+1) f_{i}^{2}-\sum_{j \in N} f_{j}^{2}\right)$

$\times\left((2 \xi-1)((n-1) \xi+1) f_{i}^{2}-\xi^{2} \sum_{j \in N} f_{j}^{2}\right)$

$\geq f_{i}^{2}((n-1) \xi+1)\left((\xi+1)((n-1) \xi+1) f_{i}^{2}-(3 \xi-1) \sum_{j \in N} f_{j}^{2}\right)$.

Denote $E=((n-1) \xi+1) f_{i}^{2}$ and $F=\sum_{j \in N} f_{j}^{2}$ and the condition simplifies to:

$$
\begin{aligned}
& (E-F)\left((2 \xi-1) E-\xi^{2} F\right) \geq E((\xi+1) E-(3 \xi-1) F) \\
\Rightarrow & (2 \xi-1) E^{2}-\xi^{2} E F-(2 \xi-1) E F+\xi^{2} F^{2} \\
& \geq(\xi+1) E^{2}-(3 \xi-1) E F \\
\Rightarrow & (\xi-2) E^{2}-\left(\xi^{2}-\xi\right) E F+\xi^{2} F^{2} \geq 0 \\
\Rightarrow & (\xi F-(\xi-2) E)(\xi F-E) \geq 0 .
\end{aligned}
$$

By Proposition 2, $\xi F-E>0$. Thus we must have:

$$
\xi \sum_{j \in N} f_{j}^{2}-(\xi-2)((n-1) \xi+1) f_{i}^{2} \geq 0 \text {. }
$$

For $\xi<1$,

$$
\begin{aligned}
& (E-F)\left((2 \xi-1) E-\xi^{2} F\right) \leq E((\xi+1) E-(3 \xi-1) F) \\
\Rightarrow & (2 \xi-1) E^{2}-\xi^{2} E F-(2 \xi-1) E F+\xi^{2} F^{2} \\
& \leq(\xi+1) E^{2}-(3 \xi-1) E F \\
\Rightarrow & (\xi-2) E^{2}-\left(\xi^{2}-\xi\right) E F+\xi^{2} F^{2} \leq 0 \\
\Rightarrow & (\xi F-(\xi-2) E)(\xi F-E) \leq 0 .
\end{aligned}
$$

Again by Proposition 2, $\xi F-E<0$. Thus we must have:

$$
\xi \sum_{j \in N} f_{j}^{2}-(\xi-2)((n-1) \xi+1) f_{i}^{2} \geq 0,
$$

which is same as what get for $\xi>1$.

\section{References}

Aksoy, Y., \& Erengüç, S. (1988). Multi-item models with coordinated replenishments: a survey. International Journal of Production Management, 8, 63-73.

Anily, S., \& Haviv, M. (2007). The cost allocation problem for the first order interaction joint replenishment model. Operations Research, 55, 292-302.

Bauso, D., Giarre, L., \& Presenti, R. (2008). Consensus in noncooperative dynamic games: a multiretailer inventory application.. IEEE Transactions on Automatic Control, 53, 998-1003.

Fiestras-Janeiro, M., Garcia-Jurado, I., Meca, A., \& Mosquera, M. (2011). Cooperative game theory and inventory management. European Journal of Operational Research, 210, 459-466.

Fiestras-Janeiro, M., García-Jurado, I., Meca, A., \& Mosquera, M. (2015). Cooperation on capacitated inventory situations with fixed holding costs. European Journal of Operational Research, 241, 719-726.

Gilbert, H. (2010). BMW and Daimler set to expand joint procurement. http:// www.supplymanagement.com/news/2010/bmw-and-daimler-set-to-expandjoint-procurement/.

Harris, F. W. (1913). How many parts to make at once. Factory, The Magazine of Management, 10, 135-136, 152.

Hartman, B., \& Dror, M. (2007). Shipment consolidation: who pays for it and how much? Management Science, 53, 78-87.

Jans, R., \& Degraeve, Z. (2008). Modeling industrial lot sizing problems: a review. International Journal of Production Research, 46, 1619-1643.

Khouja, M., \& Goyal, S. (2008). A review of the joint replenishment problem literature: 1989-2005. European Journal of Operational Research, 86, 1-16.

Körpeoğlu, E., Şen, A., \& Güler, K. (2012). A private contributions game for joint replenishment. Mathematical Methods of Operations Research, 75, 67-82.

Körpeoğlu, E., Sen, A., \& Güler, K. (2013). Non-cooperative joint replenishment under asymmetric information. European Journal of Operational Research, 277, 434-443.

Meca, A., Garcia-Jurado, I., \& Borm, P. (2003). Cooperation and competition in inventory games. Mathematical Methods of Operations Research, 57, 481-493.

Meca, A., Timmer, J., Garcia-Jurado, I., \& Borm, P. (2004). Inventory games. European Journal of Operational Research, 156, 127-139.

Minner, S. (2007). Bargaining for cooperative economic ordering. Decision Support Systems, 43, 569-583.

Myerson, R. B., \& Satterthwaite, M. A. (1983). Efficient mechanisms for bilateral trading. Journal of Economic Theory, 29, 265-281.

Speranza, M. G. (2016). Trends in transportation and logistics. European Journal of Operational Research. Available online http://dx.doi.org/10.1016/j.ejor.2016.08. 032.

Timmer, J., Chessa, M., \& Boucherie, R. J. (2013). Cooperation and game-theoretic cost allocation in stochastic inventory models with continuous review. European Journal of Operational Research, 231, 567-576.

Williams, S. R. (1999). A characterization of efficient, bayesian incentive compatible mechanisms. Economic Theory, 14, 155-180.

Zhang, J. (2009). Cost allocation for joint replenishment models. Operations Research, 57, 146-156.

Zipkin, P. H. (2000). Foundations of Inventory Management. New York: McGraw-Hill Higher Education. 